\title{
Fracturas de la columna vertebral en pacientes con espondilitis anquilosante
}

\author{
Pedro L. Bazán,,",", Martín A. Bravo,,"\# Edgar E. Gutiérrez, ${ }^{*}$ Sergio Terraza, ${ }^{\not z}$ Carlos Cortés, ${ }^{*}$ Álvaro E. Borri,,"* \\ Martín Medina, ,"* Nicolás M. Ciccioli,$* *$ \\ "Unidad de Patología Espinal, Hospital Interzonal General de Agudos “José de San Martín”, La Plata, Buenos Aires, Argentina \\ **Hospital Italiano La Plata, Buenos Aires, Argentina \\ "Instituto de Diagnóstico La Plata, Buenos Aires, Argentina \\ \#Hospital Municipal de Agudos “Leónidas Lucero”, Bahía Blanca, Buenos Aires, Argentina \\ ${ }^{*}$ Instituto de Ortopedia y Traumatología, Posadas, Misiones, Argentina \\ ${ }^{*}$ Clínica Francesa, Mendoza, Argentina \\ ${ }^{\ddagger}$ Instituto Traumatológico, Santiago de Chile, Chile \\ ${ }^{\sharp}$ Clínica Privada Pueyrredón, Mar del Plata, Buenos Aires, Argentina
}

\section{RESUMEN}

Introducción: La espondilitis anquilosante es un trastorno inflamatorio progresivo que afecta el esqueleto axial, inclusive las articulaciones sacroilíacas; el riesgo de sufrir una fractura se cuadruplica (10\% a los 10 años de enfermedad), la tasa de demora del diagnóstico es alta. La rigidez y la osteoporosis son factores clave para sufrir estas lesiones. La tomografía computarizada y la resonancia magnética cumplen un rol diagnóstico fundamental. La descompresión y la fijación quirúrgica es el tratamiento de elección actual. Se presenta una serie de casos con el objetivo de considerar las dificultades diagnósticas, describir las lesiones y la decisión terapéutica, analizar la presentación de complicaciones y realizar una actualización bibliográfica. Materiales y Métodos: Estudio multicéntrico retrospectivo de una serie de casos con 6 pacientes. Resultados: Seis hombres, edad promedio 58.1 años. Cuatro habían sufrido una caída desde la posición de pie. El tiempo promedio hasta el diagnóstico fue de 12.8 días. Los sectores más afectados fueron el torácico y el lumbar, con un mecanismo predominante en hiperextensión. Cuatro pacientes recibieron tratamiento quirúrgico. Conclusiones: Los pacientes con espondilitis anquilosante tienen un riesgo más alto de sufrir una fractura por traumas de baja energía. La demora para llegar al diagnóstico fue de 12.8 días. La cirugía con fijaciones largas y liberación por vía posterior es el tratamiento más utilizado. No se observaron complicaciones después del tratamiento.

Palabras clave: Espondilitis anquilosante; fracturas; tratamiento; complicaciones.

Nivel de Evidencia: IV

\section{Spine Fractures in Patients with Ankylosing Spondylitis}

\section{ABSTRACT}

Introduction: Ankylosing Spondylitis (AS) is a progressive inflammatory disorder that affects the axial skeleton including the sacroiliac joints. Patients are 4 times more likely to suffer a fracture (10\% at 10 years of illness), and there is a high percentage of delay in diagnosis. Stiffness and osteoporosis are key to suffering these injuries. CT and MRI scannings play a fundamental role in diagnosis. The current choice for treatment is decompression and surgical fixation. A series of cases is presented in order to: consider diagnostic difficulties; describe the injuries and therapeutic decision; analyze the presentation of complications and carry out a bibliographic update. Materials and Methods: This is a retrospective multicenter study of a case series of 6 patients. Results: Six males with an average age of 58.1 years. Four presented a fall from the standing position. The delay in diagnosis was 12.8 days on average. The most affected areas were thoracic and lumbar, with a predominant mechanism of hyperextension. Four patients underwent surgery. Conclusions: AS carriers are more at risk of suffering a low-energy trauma fracture. A delay of 12.8 days in diagnosis. Surgical treatment, with long fixations and posterior release, is the most widely used treatment. We have not observed post treatment complications.

Key words: Ankylosing spondylitis; fractures; treatment; complications.

Level of Evidence: IV 


\section{INTRODUCCIÓN}

La espondilitis anquilosante (EA) es un trastorno inflamatorio progresivo que afecta principalmente el esqueleto axial, inclusive las articulaciones sacroilíacas. ${ }^{1}$ Los pacientes con EA tienen cuatro veces más riesgo de sufrir una fractura durante la etapa más activa de su vida que la población general, ${ }^{2,3}$ con una prevalencia del $10 \%$ luego de 10 años de enfermedad, ${ }^{4}$ teniendo en cuenta, además, que estas fracturas pueden ser clínicamente indetectables cuando el paciente ingresa. ${ }^{5-7}$ En la actualidad, se considera a la osteoporosis como una característica común de la EA, incluso en las primeras etapas de la enfermedad, ${ }^{8}$ con una tasa de prevalencia del $19 \%$ y al $61 \% .{ }^{9}$ La columna cervical seguida de la columna torácica son los sitios más frecuentes de lesión. ${ }^{10-12}$ La radiografía ofrece poca ayuda para el diagnóstico de fracturas vertebrales. La tomografía computarizada (TC) y la resonancia magnética (RM) brindan más detalles sobre los segmentos fracturados. ${ }^{1}$ Las fracturas cervicales tienden a ser muy inestables y pueden provocar un daño neurológico secundario. ${ }^{13,14}$ El tratamiento conservador se ha correlacionado con una tasa de mortalidad alta; ${ }^{13}$ por lo tanto, actualmente, el manejo quirúrgico de estas enfermedades es de elección, incluidas la descompresión, la reducción de los trazos fracturarios y la fusión instrumentada. ${ }^{15}$

Se presenta una serie de casos con el objetivo de evaluar la cinemática, considerar las dificultades diagnósticas, describir las lesiones y las posibilidades terapéuticas, analizar la presentación de complicaciones y realizar una actualización bibliográfica.

\section{MATERIALES Y MÉTODOS}

Se realizó un análisis retrospectivo de seis pacientes con diagnóstico de EA que sufrieron fracturas de la columna vertebral luego de un traumatismo, y fueron tratados en cinco centros diferentes (4 de Argentina y 1 de Chile). A todos se los evaluó mediante radiografías simples, TC y RM.

\section{RESULTADOS}

Todos los pacientes eran hombres, con una media de la edad de 58.1 años (rango 40-77). Las lesiones se habían producido por un accidente automovilístico en dos pacientes (un caso en auto con cinturón, choque lateral y otro en motocicleta, con una fractura de fémur asociada) y por una caída en posición de pie en cuatro pacientes.

El tiempo promedio hasta el diagnóstico fue de 12.8 días (rango 0-22) y estuvo relacionado con malas imágenes radiográficas o inadecuada evaluación clínica inicial por desestimar el dolor ante la ausencia de déficit neurológico.

Los sectores más afectados fueron la columna torácica y lumbar (Figura 1). Las lesiones fueron clasificadas como: A1 (2 pacientes), B2 (1 paciente), B3 (1 paciente) (Figura 2) y C (2 pacientes) (mediante un mecanismo de lesión de hiperextensión) (Figura 3). Un solo paciente tenía una lesión medular completa (Frankel A o N4) al ingresar, los demás no tenían déficit sensitivo motor (N0) (Tabla).

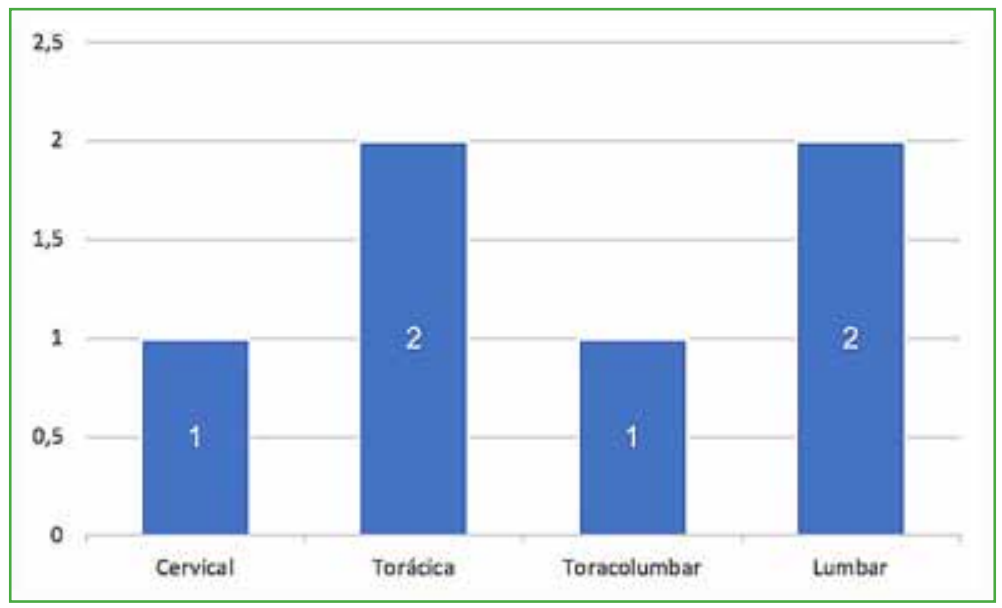

Figura 1. Distribución topográfica de las lesiones vertebrales. 


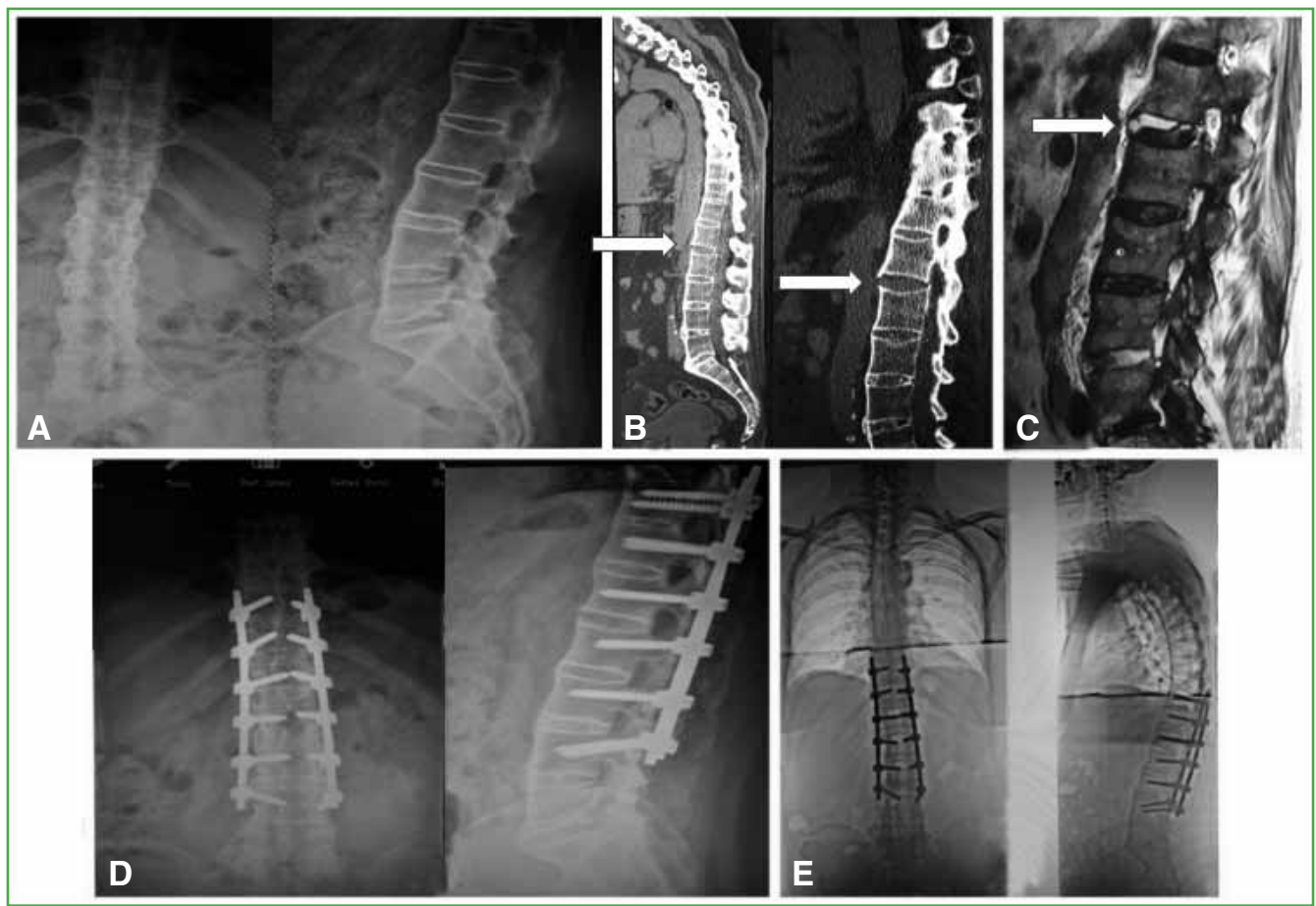

Figura 2. Paciente 5. Hombre de 57 años que sufre una caída desde la posición de pie. A. Radiografías de frente y de perfil. B. Reconstrucción sagital tomográfica, a los 15 días de la caída. C. Resonancia magnética. D. Radiografías posoperatorias. E. Espinograma a los 6 meses de la cirugía. La flecha en las imágenes B y C marca la lesión en hiperextensión.

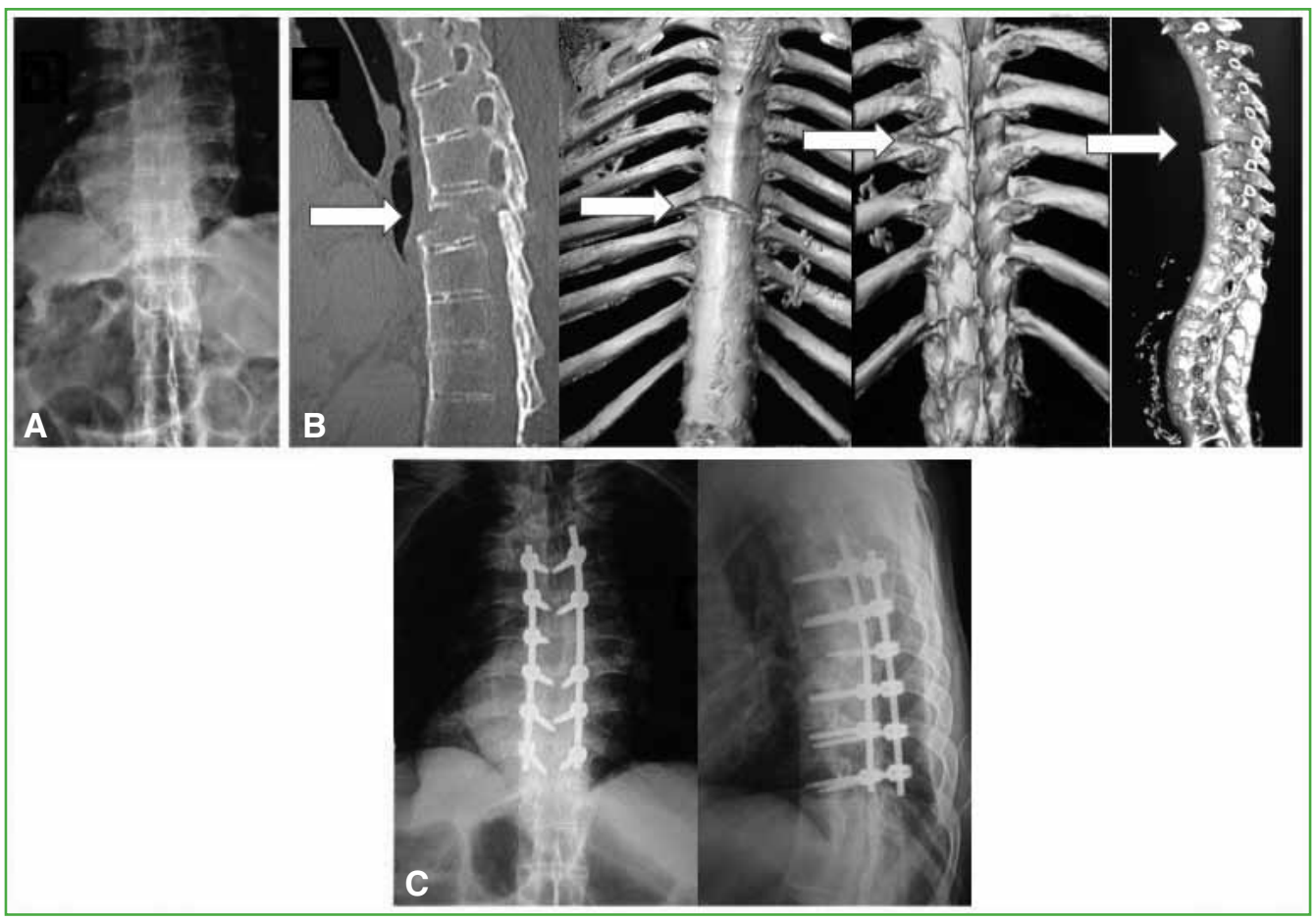

Figura 3. Paciente 2. Hombre de 77 años que sufre una caída desde la posición de pie. A. Radiografía de frente a las 48 h de la caída. B. Reconstrucción sagital tomográfica y tridimensional, 22 días después del trauma. C. Radiografías posoperatorias. La flecha marca la lesión en hiperextensión. 
Tabla. Características clínicas de los pacientes

\begin{tabular}{|c|c|c|c|c|c|}
\hline Paciente & $\begin{array}{l}\text { Edad } \\
\text { (años) }\end{array}$ & $\begin{array}{l}\text { Tipo de fractura } \\
\text { (AO) }\end{array}$ & Cinemática & $\begin{array}{c}\text { Demora del diagnóstico } \\
\text { (días) }\end{array}$ & Tratamiento \\
\hline 1 & 66 & $\begin{array}{c}\text { C6C7 B2 (C7A1) } \\
\text { N0 M3 }\end{array}$ & $\begin{array}{l}\text { Accidente } \\
\text { en auto }\end{array}$ & 22 & $\begin{array}{l}\text { Quirúrgico por vía } \\
\text { posterior }\end{array}$ \\
\hline 2 & 77 & T7T8 C N0 M2 & $\begin{array}{l}\text { Caída de propia } \\
\text { altura }\end{array}$ & 22 & $\begin{array}{l}\text { Quirúrgico por vía } \\
\text { posterior }\end{array}$ \\
\hline 3 & 67 & T6 A1 N0 M2 & $\begin{array}{l}\text { Caída de propia } \\
\text { altura }\end{array}$ & 11 & Ortopédico \\
\hline 4 & 42 & L2L3 C N4 M2 & $\begin{array}{l}\text { Accidente en } \\
\text { moto }\end{array}$ & 0 & $\begin{array}{l}\text { Quirúrgico por vía } \\
\text { posterior }\end{array}$ \\
\hline 5 & 57 & L1L2 B3 N0 M2 & $\begin{array}{l}\text { Caída de propia } \\
\text { altura }\end{array}$ & 15 & $\begin{array}{l}\text { Quirúrgico por vía } \\
\text { posterior }\end{array}$ \\
\hline 6 & 40 & L1 A1 N0 M2 & $\begin{array}{c}\text { Caída de propia } \\
\text { altura }\end{array}$ & 7 & Ortopédico \\
\hline
\end{tabular}

Cuatro fueron sometidos a una artrodesis instrumentada por vía posterior con fijación larga de más de dos niveles por encima y tres por debajo, y los dos restantes fueron tratados mediante una ortesis.

Se documentó un seguimiento promedio de tres años (rango de 10 meses a 11 años y 7 meses).

No se observaron complicaciones directas o indirectas relacionadas luego del tratamiento y la enfermedad de base evolucionó de manera habitual.

\section{DISCUSIÓN}

Las fracturas vertebrales asociadas a tasas más altas de osteoporosis y mala calidad ósea son complicaciones frecuentes en pacientes con EA. ${ }^{4}$ La posibilidad de sufrir una fractura por un traumatismo de baja energía es alta ${ }^{16}$ y la hiperextensión es el mecanismo de lesión más frecuente. ${ }^{15}$ La rigidez espinal y la baja densidad mineral ósea son las causas intrínsecas que favorecen la lesión. Los factores de riesgo mecánicos, como la fusión espinal, la disminución de la movilidad de la columna vertebral y la presencia de sindesmofitos, predisponen a sufrir fracturas vertebrales..$^{17-20}$

En los pacientes con EA, se recomienda la detección de osteoporosis dentro de los 10 años del diagnóstico y se debe iniciar el tratamiento de la osteoporosis cuando los pacientes tienen un puntaje $\mathrm{T} \leq 2,5 .{ }^{4}$ Los antinflamatorios no esteroides, el pilar del tratamiento contra la EA, pueden disminuir el riesgo de fracturas vertebrales retrasando la pérdida ósea y la fusión, y mejorando la movilidad. ${ }^{8,21,22}$

La TC se usa habitualmente para determinar el sitio de la fractura, el tipo y la gravedad. La RM se emplea para determinar los trazos fracturarios no detectables con la TC (edema óseo), reconocer una posible compresión de la médula espinal y determinar el rango exacto de descompresión necesaria, en caso de cirugía..$^{23}$ Las complicaciones, como lesiones de la médula espinal, lesiones de la raíz nerviosa y el hematoma paravertebral, son siempre más frecuentes y más graves en pacientes con EA. ${ }^{23}$

En un metanálisis extenso, Westerveld y cols. ${ }^{2}$ señalaron que el tratamiento quirúrgico llevó a una mejoría neurológica y una tasa de complicaciones global inferior a la del tratamiento no quirúrgico tanto a corto como a largo plazo. El objetivo principal de la cirugía es mantener realineada la fractura con una adecuada estabilización hasta que se logre la consolidación. ${ }^{24}$ Los principales métodos quirúrgicos son: fusión instrumentada por vía anterior, posterior o combinada, asociada a gestos de descompresión, si es necesario. ${ }^{23}$ La estabilización anterior sola no proporciona resistencia contra la tensión de la columna posterior, lo que causa un aflojamiento, colapso del injerto óseo y aflojamiento o rotura de los fijadores internos asociados a tasas de fracaso más altas. ${ }^{25}$ Con el fin de evitar esto, se recomienda la estabilización posterior para tratar a pacientes con fracturas inestables que tienen riesgo de traslación. ${ }^{26}$ Para la mayoría de los pacientes con EA y fracturas de la columna vertebral, la fijación debe realizarse tres segmentos por encima y por debajo del cuerpo vertebral lesionado, lo que permitiría lograr una buena 
eficacia clínica. ${ }^{23}$ Las fracturas cervicales son un cuadro muy inestable que comúnmente causa déficit neurológico progresivo. ${ }^{13,14}$ Luksanapruksa y cols. compararon los resultados quirúrgicos del abordaje posterior y los abordajes combinados para la reparación de fracturas cervicales en pacientes con EA y comunicaron que tanto el abordaje posterior como los abordajes combinados obtuvieron buenos resultados, incluida la mejoría del estado neurológico sin la necesidad de reoperación. ${ }^{15}$ Longo y cols. ${ }^{27}$ en una revisión sistemática de 2015 , mostraron que las fracturas cervicales eran más comunes en los hombres (94\%) y que típicamente ocurrían en pacientes de mediana edad (media de la edad 59.2 años). Los niveles afectados fueron C6-7, C5-6 y C4-5. La mayoría tuvo una mejoría neurológica posoperatoria de, al menos, 1 grado de Frankel (78\% con abordaje combinado, 79\% con abordaje anterior y $70 \%$ con abordaje posterior). La tasa de complicaciones médicas posoperatorias fue del $19 \%$ (14\% con el abordaje anterior, $26 \%$ con el abordaje posterior y $16 \%$ con el abordaje combinado), mientras que la tasa de complicaciones intraoperatorias fue del 16\% (14\% con el abordaje anterior, $15 \%$ con el abordaje posterior y $18 \%$ con el abordaje combinado). ${ }^{27}$

\section{CONCLUSIONES}

Los pacientes con EA corren más riesgo de sufrir una fractura vertebral por traumatismo de baja energía que la población general. Es importante tener un alto nivel de sospecha ante un paciente con EA y un antecedente traumático que solo sufra dolor, para evitar las complicaciones neurológicas. En nuestro estudio, el promedio de días hasta el diagnóstico fue 12 y se relacionó con la falta de estudios o la mala calidad de estos.

En esta serie, el mecanismo de lesión más frecuente fue la hiperflexión con la consiguiente lesión de la banda de tensión posterior asociada o no con traslación.

Los antinflamatorios no esteroides brindarían un efecto protector frente al riesgo de sufrir una fractura vertebral en pacientes con EA. El tratamiento quirúrgico con fijaciones largas y liberación por vía posterior es el más utilizado. En nuestra serie, no se observaron complicaciones posquirúrgicas. Se cree necesaria una muestra con más pacientes.

Conflicto de intereses: Los autores no declaran conflicto de intereses.

ORCID de M. A. Bravo: https://orcid.org/0000-0003-0848-4823 ORCID de E. E. Gutiérrez: https://orcid.org/0000-0002-8859-0919 ORCID de S. Terraza: https://orcid.org/0000-0002-2807-1690 ORCID de C. Cortés: https://orcid.org/0000-0002-0571-2547
ORCID de Á. E. Borri: https://orcid.org/0000-0002-5568-867X ORCID de M. Medina: https://orcid.org/0000-0002-5281-5645 ORCID de N. M. Ciccioli: https://orcid.org/0000-0002-5851-2821

\section{BIBLIOGRAFÍA}

1. Leone A, Marino M, Dell'Atti C, Zecchi V, Magarelli N, Colosimo C. Spinal fractures in patients with ankylosing spondylitis. Rheumatol Int 2016;36(10):1335-46. https://doi.org/10.1007/s00296-016-3524-1

2. Westerveld LA, Verlaan JJ, Oner FC. Spinal fractures in patients with ankylosing spinal disorders: a systematic review of the literature on treatment, neurological status and complications. Eur Spine J 2009;18(2):145-56. https://doi.org/10.1007/s00586-008-0764-0

3. Finkelstein JA, Chapman JR, Mirza S. Occult vertebral fractures in ankylosing spondylitis. Spinal Cord 1999;37(6):444-7. https://doi.org/10.1038/sj.sc.3100837

4. Davey-Ranasinghe N, Deodhar A. Osteoporosis and vertebral fractures in ankylosing spondylitis. Curr Opin Rheumatol 2013;25:509-16. https://doi.org/10.1097/BOR.0b013e3283620777

5. Jacobs WB, Fehlings MG. Ankylosing spondylitis and spinal cord injury: origin, incidence, management, and avoidance. Neurosurg Focus 2008;24(1):E12. https://doi.org/10.3171/FOC/2008/24/1/E12

6. Waldman SK, Brown C, Lopez de Heredia L, Hughes RJ. Diagnosing and managing spinal injury in patients with ankylosing spondylitis. J Emerg Med 2017;44(4):e315-e319. https://doi.org/10.1016/j.jemermed.2012.11.002

7. Sambrook PN, Geusens P. The epidemiology of osteoporosis and fractures in ankylosing spondylitis. Ther Adv Musculoskelet Dis 2012;4(4):287-92. https://doi.org/10.1186/ar3724 
8. Ghozlani I, Ghazi M, Nouijai A, Mounach A, Rezqi A, Achemlal L, et al. Prevalence and risk factors of osteoporosis and vertebral fractures in patients with ankylosing spondylitis. Bone 2009;44:772-6.

https://doi.org/10.1016/j.bone.2008.12.028

9. Vosse D, de Vlam K. Osteoporosis in rheumatoid arthritis and ankylosing spondylitis. Clin Exp Rheumatol 2009;27(4 Suppl 55):S62-S67. Disponible en: https://www.clinexprheumatol.org/article.asp?a=3710

10. Yilmaz N, Pence S, Kepekci Y, Geyikli I, Ozaslan J. Association of immune function with bone mineral density and biochemical markers of bone turnover in patients with anklylosing spondylitis. Int J Clin Pract 2003;57:681-5. PMID: 14627178

11. El Maghraoui A, Bensabbah R, Bahiri R, Bezza A, Guedira N, Hajjaj-Hassouni N. Cervical spine involvement in ankylosing spondylitis. Clin Rheumatol 2003;22:94-8. https://doi.org/10.1007/s10067-002-0681-2

12. Longo UG, Loppini M, Denaro L, Maffulli N, Denaro V. Conservative management of patients with an osteoporotic vertebral fracture: a review of the literature. J Bone Joint Surg Br 2012;94:152-7. https://doi.org/10.1302/0301-620X.94B2.26894

13. Altenbernd J, Bitu S, Lemburg S, Peters S, Seybold D, Meindl R, et al. Vertebral fractures in patients with ankylosing spondylitis: a retrospective analysis of 66 patients. Rofo 2009;181:45-53. https://doi.org/10.1055/s-2008-1027886

14. Caron T, Bransford R, Nguyen Q, Agel J, Chapman J, Bellabarba C. Spine fractures in patients with ankylosing spinal disorders. Spine 2010;35:E458-64. https://doi.org/10.1097/BRS.0b013e3181cc764f

15. Luksanapruksa P, Millhouse PW, Carlson V, Ariyawatkul T, Heller J, Kepler CK. Comparison of surgical outcomes of the posterior and combined approaches for repair of cervical fractures in ankylosing spondylitis. Asian Spine $J$ 2019;13(3):432-40. https://doi.org/10.31616/asj.2018.0197

16. Pray C, Feroz NI, Nigil Haroon N. Bone mineral density and fracture risk in ankylosing spondylitis: a metaanalysis. Calcif Tissue Int 2017;101(2):182-92. https://doi.org/10.1007/s00223-017-0274-3

17. Montala N, Juanola X, Collantes E, Munoz-Gomariz E, Gonzalez C, Gratacos J, et al. Prevalence of vertebral fractures by semi-automated morphometry in patients with ankylosing spondylitis. J Rheumatol 2011;38:893-7. https://doi.org/10.3899/jrheum.100851

18. Donnelly S, Doyle DV, Denton A, Rolfe I, McCloskey EV, Spector TD. Bone mineral density and vertebral compression fracture rates in ankylosing spondylitis. Ann Rheum Dis 1994;53(2):117-21.

https://doi.org/10.1136/ard.53.2.117

19. Jun JB, Joo KB, Her MY, Kim TH, Bae SC, Yoo DH, et al. Femoral bone mineral density is associated with vertebral fractures in patients with ankylosing spondylitis: a cross-sectional study. J Rheumatol 2006;33(8):1637-41. PMID: 16881119

20. Klingberg E, Geijer M, Gothlin J, Mellstrom D, Lorentzon M, Hilme E, et al. Vertebral fractures in ankylosing spondylitis are associated with lower bone mineral density in both central and peripheral skeleton. J Rheumatol 2012;39:1987-95. https://doi.org/10.3899/jrheum.120316

21. Muñoz-Ortego J, Vestergaard P, Rubio JB, Wordsworth P, Judge A, Javaid MK, et al. Ankylosing spondylitis is associated with an increased risk of vertebral and non-vertebral clinical fractures: a population-based cohort study. J Bone Miner Res 2014;29(8):1770-6. https://doi.org/10.1002/jbmr.2217

22. Vosse D, Landewé R, van der Heijde D, van der Linden S, van Staa TP, Geusens P. Ankylosing spondylitis and the risk of fracture: results from a large primary care-based nested casecontrol study. Ann Rheum Dis 2009;68(12):1839-42. https://doi.org/10.1136/ard.2008.100503

23. Zhu R1, Song W, Hu W, Jiang Z, Yuan J, Cui Z, Wan J, Liu Y, Feng S, Zhang X. The treatment strategies for spine fractures in patients with ankylosing spondylitis: A case report. Medicine (Baltimore) 2017;96(44):e8462. https://doi.org/10.1097/MD.0000000000008462

24. Heyde CE, Fakler JK, Hasenboehler E, Stahel PF, John T, Robinson Y, et al. Pitfalls and complications in the treatment of cervical spine fractures in patients with ankylosing spondylitis. Patient Saf Surg 2008;2:15. https://doi.org/10.1186/1754-9493-2-15

25. Lu GH, Wang B, Li J, Kang YJ, Lu C, Ma ZM, et al. Combined anterior and posterior approach for cervical fracture-dislocation with ankylosing spondylitis. Zhonghua Wai Ke Za Zhi 2007;45:373-5. PMID: 17537319

26. Shen FH, Samartzis D. Surgical management of lower cervical spine fracture in ankylosing spondylitis. J Trauma 2006;61:1005-9. https://doi.org/10.1097/01.ta.0000208125.63010.04

27. Longo UG, Loppini M, Petrillo S, Berton A, Maffulli N, Denaro V. Management of cervical fractures in ankylosing spondylitis: anterior, posterior or combined approach? Br Med Bull 2015;115:57-66.

https//doi.org/10.1093/bmb/ldv010 